\title{
A COMPARISON OF SUPRASCAPULAR NERVE BLOCK VS INTRAARTICULAR STEROID INJECTION IN IMPROVEMENT OF HEMIPLEGIC SHOULDER PAIN
}

\author{
Musab Bin Noor, Aamir Waheed Butt*, Waseem Iqbal**, Maimuna Rashid** \\ Armed Forces Institute of Rehabilitation Medicine, Rawalpindi Pakistan, ${ }^{*}$ Combined Military Hospital Lahore/National University of Medical Sciences (NUMS) \\ Pakistan, ${ }^{* *}$ Combined Military Hospital Peshawar/National University of Medical Sciences (NUMS) Pakistan
}

\begin{abstract}
Objective: To compare the mean improvement in pain on Visual Analogue Scale by Suprascapular nerve block and Intraarticular steroid injection in patients with hemiplegic shoulder pain.

Study Design: Quasi-experimental study.

Place and Duration of Study: Interventional Pain Management Center, Armed Forces Institute of Rehabilitation Medicine Rawalpindi, from Oct 2017 to Apr 2018.

Methodology: A total of 60 patients with stroke and shoulder pain on hemiplegic side for at least 2 weeks were included, randomized by lottery into two groups; Group A receiving Suprascapular Nerve block with $5 \mathrm{ml}$ of $1 \%$ Lignocaine with Group $B$ receiving Intra-articular 40mg Triamcinolone Acetonide plus $1 \mathrm{ml}$ of $1 \%$ Lignocaine injections. Mean change in pain scores on Visual Analogue Scale was calculated between score at baseline and at 4 weeks post-intervention.

Results: Out of a total of 60 subjects, $34(56.67 \%)$ were males and $26(43.33 \%)$ were females. Mean duration of stroke was $7.50 \pm$ 2.66 months. Mean pain change in group A (Suprascapular Nerve block) was $3.83 \pm 1.12 \mathrm{~cm}$ while in group B (intra-articular steroid injection) was $2.17 \pm 0.99 \mathrm{~cm}(p$-value $<0.001)$.

Conclusion: Suprascapular Nerve block offered better improvement in hemiplegic shoulder pain on Visual Analogue Scale at 4 weeks as compared to intraarticular steroid injection.
\end{abstract}

Keywords: Hemiplegic shoulder pain, Intraarticular steroid injection, Pain management, Suprascapular nerve block.

How to Cite This Article: Noor MB, Butt AW, Iqbal W, Rashid M. A Comparison of Suprascapular Nerve Block Vs Intraarticular Steroid Injection in Improvement of Hemiplegic Shoulder Pain. Pak Armed Forces Med J 2021; 71(6): 1971-1975. D Doi: https://doi.org/10.51253/pafmj.v6i6.3589

This is an Open Access article distributed under the terms of the Creative Commons Attribution License (https://creativecommons.org/licenses/by-nc/4.0/), which permits unrestricted use, distribution, and reproduction in any medium, provided the original work is properly cited.

\section{INTRODUCTION}

Stroke or Cerebrovascular Accident (CVA) is a common and debilitating condition with an incidence of approx. $250 / 100,000$ population in Pakistan. ${ }^{1}$ It is a major life changing event for the patient and results in a number of significant impairments, most common of which is hemiplegia, affecting up to $88 \%$ of stroke survivors. $^{2}$ One of the most important complications of hemiplegia is shoulder dysfunction on the affected side, manifested as shoulder pain and restriction of movement, with frequency varying from $16-84 \%$ according to various studies. ${ }^{3,4}$

Hemiplegic shoulder dysfunction is a multifactorial pathology resulting from a disturbance in normal shoulder dynamics as a result of hemiplegia. The most commonly implicated pathologies include Supraspinatus tendon and Biceps tendon pathology, ${ }^{5}$ however other factors such as glenohumeral subluxation, adhesive capsulitis and long head of biceps tendon effusion are also implicated. Kalichman et $a l^{6}$ in a comprehen-

Correspondence: Dr Musab Bin Noor, 64 Medical Battalion, Saibzad Gul Road, Peshawar Pakistan

Received: 03 Apr 2020; revision received: 09 Oct 2020; accepted: 12 Oct 2020 sive review of Hemiplegic Shoulder Dysfunction etiologies, identified three separate classes of specific pathologies: (a) lesions involving primarily soft tissues, (b) pathological alterations in motor control (especially in resting and active muscular tone), and (c) an alteration in neurological activity of the central as well as peripheral nervous system. Importantly, the authors found that each could present as a separate entity, combine as a syndrome, or act as a trigger in each other's progression.

Hemiplegic shoulder dysfunction is one of the most important predictors of poor recovery of arm power and function and is an important predictor of hospital length of stay. Due to its multifactorial etiology, a number of treatment strategies have been formulated for its management. The focus of management of hemiplegic shoulder pain is to target the intervention at the initial phase of post-stroke shoulder dysfunction. This stage manifests with flaccidity of the shoulder musculature, and can result in injury to the soft tissues, inferior subluxation of humeral head and excessive traction on the glenohumeral joint complex. Early intervention is thought to be advisable, as contracture can occur quite rapidly. In the later stages, spasticity 
becomes prominent, resulting in severe limitation of movement. In 2000, a study was performed in Holland which revealed that the majority of treating physicians considered Intraarticular Steroid Injections as first line treatments in the management of hemiplegic shoulder pain. Unfortunately, local injection with corticosteroids has had unsatisfactory results in a large number of hemiplegic shoulder pain patients unless they are used to specifically target an inflammatory cause of hemiplegic shoulder pain, e.g. rotator cuff tendinitis, biceps tendinitis, adhesive capsulitis or subacromial bursitis. There has been modest improvement in pain with subacromial rather than intraarticular Steroid injection, which is likely an indication that rotator cuff impingement or tendonitis was the primary pathologic process of hemiplegic shoulder pain.

Intra-articular steroid injections and Supra-scapular nerve blocks are commonly used to varying effects in patients with shoulder pain, with Suprascapular nerve blocks resulting in improvements from a baseline Visual analogue scale (VAS) score of 81 (71.06$9.094)$ to a score of $35.5 \pm 11.4$ four weeks after intervention, ${ }^{8}$ and intraarticular steroid injection showing improvement from baseline VAS score of 5.5 (3.8-7.2) to $3.6 \pm 2.0$ four weeks after intervention. ${ }^{9} \mathrm{~A}$ few pilot studies have been carried out internationally to compare the effects of both these techniques in the hemiplegic population. ${ }^{10}$ There have also been a number of studies comparing the effect of suprascapular nerve block and intra-articular steroid on shoulder pain in non-hemiplegic populations in Pakistan, however there is need for comparing the efficacy of these two techniques in the Pakistani hemiplegic population.

The rationale of this study was to fill the gaps in local literature on interventional procedures in the management of hemiplegic shoulder pain. Results from this study will guide the management of a large population of affected patients reporting to Rehabilitation based tertiary care hospitals.

\section{METHODOLOGY}

The patients were recruited from the outpatient Stroke Clinic at AFIRM. The study was carried out at the AFIRM Pain Clinic, a center where interventional pain management procedures are carried out on inpatient and outpatient population. After receiving approval from the institutional ethics review committee for initiation of a quasi-experimental study, 60 cases were recruited using non-probability consecutive sampling (Hospital Ethical Review Committee letter ref no. 06/Trg/AFIRM date 24/02/2020). The sample size was calculated using WHO Sample Size calculator.

Inclusion Criteria: Patients of both genders, age ranged from 18-80 years, with a diagnosis of cerebrovascular accident in the last 12 months were included. In addition the presence of hemiplegia as a stroke sequela and complaints of localized passive or active shoulder pain with a minimum Visual Analogue Scale score of $40 \mathrm{~mm}(4 \mathrm{~cm})$ or higher out of $100 \mathrm{~mm}(10 \mathrm{~cm})$ on affected side for at least 2 weeks were also included.

Exclusion Criteria: Any patient with cognitive deficits that precluded them from being able to reliably use subjective scales of outcome measurement (MiniMental State Examination (MMSE) <23) were excluded from the study, as were patients with known hypersensitivity to injected agents. Patients with contraindications to Steroid use including Septic Arthritis, local skin infection and uncontrolled hyperglycemia were also excluded. Previous history of trauma or shoulder pain on affected side as well as previous history of Intra-Articular Injection or Nerve Block in affected shoulder were also considered for exclusion criteria.

Patients' informed consent for participation and follow up was taken after the objectives and benefits of the study were explained in detail. The study subjects were randomized by lottery into two groups; group A receiving Suprascapular Nerve blocks with $5 \mathrm{ml}$ of $1 \%$ Lignocaine with group B receiving Intra-articular 40 mg Triamcinolone Acetonide plus 1ml 1\% Lignocaine injections in the affected shoulder. Both groups underwent interventional procedures using aseptic technique without the use of imaging modalities.

Demographic data including age, gender and duration since stroke, type of stroke (ischemic v/s hemorrhagic) and side affected by hemiplegia were recorded while pain severity was measured on Visual Analogue Scale pre-intervention and at 4-week interval. The mean change in pain scores on VAS was calculated between VAS score at baseline and at 4 weeks post-intervention. Recorded data was analyzed by utilizing Statistical Package for Social Science IBM (SPSS) version 23. Frequencies along with percentages were calculated for categorical (qualitative) variables including gender, type of stroke and affected side. Descriptive statistics i.e., mean and SD were calculated for continuous (quantitative) variables including age, duration since stroke and pain values on VAS. Student T-test was applied to see the difference in mean pain change between the two groups. The $p$-value $\leq 0.05$ was considered as significant. 


\section{RESULTS}

All 60 patients reported for follow up after 4 weeks, with no drop outs. Age range in this study was from 18 to 80 years with mean age of $60.06 \pm 9.91$ years. The mean age of patients in group A was $59.17 \pm 9.81$ years and in group B was $60.96 \pm 11.11$ years (Table-I). Majority of the patients (47) (78.33\%) were between 51 to 80 years of age. Out of 60 patients, $34(56.67 \%)$ were males and $26(43.33 \%)$ were females. Mean duration since stroke was $7.50 \pm 2.66$ months. The mean duration of stroke in group A was $7.33 \pm 2.63$ months and in group B was $7.67 \pm 2.72$ months as shown in Table-II. Distribution of patients with respect to type of stroke is shown in Table-III. Mean pain change in Group A (suprascapular nerve block) was $3.83 \pm 1.12 \mathrm{~cm}$ while in group B (Intra-articular steroid injection) was $2.17 \pm$ $0.99 \mathrm{~cm}$ as shown in Table-IV ( $p$-value $<0.001$ ).

Table-I: Age distribution for both groups $(n=60)$.

\begin{tabular}{l|c|c}
\hline \multirow{2}{*}{ Age (years) } & Group A (n=30) & Group B (n=30) \\
\cline { 2 - 3 } & $\mathbf{n ~ ( \% )}$ & $\mathbf{n}(\mathbf{0})$ \\
\hline $18-50$ & $6(20 \%)$ & $7(23.33 \%)$ \\
\hline $51-80$ & $24(80 \%)$ & $23(76.67 \%)$ \\
\hline Mean \pm SD & $59.17 \pm 9.81$ & $60.96 \pm 11.11$ \\
\hline
\end{tabular}

Table-II: Distribution of patients according to duration of disease $(n=60)$.

\begin{tabular}{l|c|c}
\hline $\begin{array}{l}\text { Duration of } \\
\text { disease (months) }\end{array}$ & Group A (n=30) & Group B (n=30) \\
\cline { 2 - 3 }$\leq 6$ months & $\mathbf{n ~ ( \% )}$ & $\mathbf{n ~ ( \% )}$ \\
\hline $7-12$ months & $13(43.33 \%)$ & $10(33.33 \%)$ \\
\hline Mean \pm SD & $77(56.67 \%)$ & $20(66.67 \%)$ \\
\hline
\end{tabular}

Table-III: Distribution of patients according to type of stroke $(n=60)$.

\begin{tabular}{l|c|c|c}
\hline \multirow{2}{*}{ Type of Stroke } & Group A (n=30) & Group B (n=30) \\
\cline { 2 - 4 } & $\mathbf{n ~ ( \% )}$ & $\mathbf{n ~ ( \% )}$ \\
\hline Ischemic & $19(63.33 \%)$ & $15(50 \%)$ \\
\hline Hemorrhagic & $11(36.67 \%)$ & $15(50 \%)$ \\
\hline Table-IV: Change in pain scores on visual analog scale. \\
\hline Group & $\begin{array}{c}\text { Group A } \\
(\mathbf{n}=\mathbf{3 0 )}\end{array}$ & $\begin{array}{c}\text { Group B } \\
(\mathbf{n}=\mathbf{3 0 )}\end{array}$ & $\begin{array}{c}\boldsymbol{p} \text { - } \\
\text { value }\end{array}$ \\
\hline $\begin{array}{l}\text { Change in Pain } \\
\text { Score (Mean } \pm \text { SD) }\end{array}$ & $3.83 \pm 1.12$ & $2.17 \pm 0.99$ & $<0.001$ \\
\hline
\end{tabular}

\section{DISCUSSION}

Hemiplegic shoulder pain is one of the commonest morbidities occurring in the post-stroke population, occurring in up to $70 \%$ of patients 3 and often appearing in the early 'flaccid' post-stroke stage. It is one of the markers of stroke severity, with up to $75 \%$ of patients complaining of shoulder pain in the affected side at one time or another in the first 52 weeks after a stroke. All the subjects included in our study had similarly developed hemiplegic shoulder pain within 12 months of onset of stroke symptoms. The exact mec- hanisms involved in the development of hemiplegic shoulder pain are often unclear but as the upper limb is primarily attached to the torso by a muscular connection rather than direct skeletal interface at glenohumeral joint, any dysfunction that primarily affects the action of the muscles can result in biomechanical disruptions, subsequently resulting in shoulder pain. Hemiplegic shoulder dysfunction is associated with reduction in strength of pincer grip, three jaw chuck grip and shoulder elevation, as well as development of abnormal muscle tone. There is also a strong correlation with sensory loss and the development of sensory inattention. ${ }^{12}$ Patients with hemiplegic shoulder dysfunction have impaired movement of the shoulder as well as a specific pathological posturing. This posture occurs in the setting of increased muscle tone, and consists of internal rotation, flexion and adduction.

The list of prescribed treatments for hemiplegic shoulder pain is extensive. These include rest, non-steroidal anti-inflammatory drugs (NSAIDs), active and passive mobilization, physiotherapy, intra articular corticosteroids, hydro dilatation, manipulation under anesthesia, arthroscopic capsular release, intra-articular hyaluronate injection and regional nerve block etc., with varying results. ${ }^{13-15}$

Intra-articular steroid injections and Supra-scapular nerve blocks are two of the most commonly used interventions, with international studies demonstrating suprascapular nerve blocks resulting in improvements from a baseline VAS score of $81.0 \pm 9.94$ to a score of $35.5 \pm 11.4$ four weeks after intervention, ${ }^{16}$ and intraarticular steroid injection showing improvement from baseline VAS score of $5.5+1.7$ to $3.6 \pm 2.0$ four weeks after intervention. ${ }^{17}$ The results of our study are in line with these international studies, showing both suprascapular nerve block and intraarticular steroid injection resulting in reduction of hemiplegic shoulder pain, with greater pain relief observed after Suprascapular Nerve block. Suprascapular nerve blocks have become one of the mainstays of the interventional management of persistent shoulder pain due to arthritic shoulder disease, chronic rotator cuff lesions and recurrent adhesive capsulitis. ${ }^{18,19}$ Due to its role as one of the primary sensory enervators of the shoulder joint, the SSN is a logical target for hemiplegic shoulder pain treatments. However, the results of recent international studies have proven inconclusive. Lee et al, ${ }^{20}$ reported that the efficacy of suprascapular nerve block in hemiplegic shoulder pain was unsatisfactory and failed to provide complete pain relief. More recent studies how- 
ever, have shown that suprascapular nerve block is an uncomplicated, cheap technique with very limited side effects that shows good efficacy in the management of shoulder pain. In our study, we also found Suprascapular Nerve block to be a simple procedure that can be performed in the outpatient setting, with no significant side effects observed in any of our patients. Yasar et al, ${ }^{21}$ made a comparison of Visual Analog Scale measurements for subjects who received intra-articular steroid injections or suprascapular nerve blocks. Their results suggested that both interventions effectively reduced hemiplegic shoulder pain. These results should however be interpreted carefully, as lesions with predominant inflammatory pathology will show good response to both treatments, while other hemiplegic shoulder pain etiologies will not. As suprascapular nerve blocks have a lesser side effect profile, physicians could confidently and safely employ them in management hemiplegic shoulder pain as well as in understanding its etiology.

In a 2007 retrospective study by Ben-Aviv et al at Stanford University, Palo Alto, California, the effectiveness of SSNB in the management of rotator cuff tendonitis, $88 \%$ of the subjects reported substantial and long lasting improvement in pain. The authors of the study suggested that SSNB is a less harmful intervention as compared to intraarticular steroids. ${ }^{22}$ Comparing our results with this study, all the patients in group A (suprascapular nerve block group) experienced significant pain relief, confirming the efficacy of this procedure in hemiplegic shoulder pain. A number of studies have reported that rotator cuff tendinitis is present in almost $33 \%$ of stroke survivors with hemiplegia, ${ }^{23,24}$ Hence, it is considered prudent to avoid multiple intraarticular steroid injections in hemiplegic patients, especially as they are known to cause atrophy in tendons and ligaments. A comparison of intraarticular steroid injection versus suprascapular nerve block for non-specific shoulder pain reported that both interventions resulted in improvement in pain scores, with negligible difference in terms of overall efficacy. Better results were seen however; when both interventions were combined. ${ }^{25}$ This is in contrast with the findings of our study, where although both approaches were efficacious, Suprascapular Nerve block was found to provide better pain relief. Follow-up studies utilizing a combination of both approaches can be carried out in the future to assess the efficacy of combination therapy. A study by Yi et al at Daejin Medical Center, Daejin, South Korea has shown that a blind approach for intraarticular injections in the shoulder has a success rate of $33-46 \%$. The difference is mainly dependent on the approach used, as well as the experience of the injecting physician. On the other hand, image guided intervention has a significantly higher success rate of $93 \%$. A blind, surface landmark-based approach was used for Suprascapular Nerve block and Intraarticular Steroid injection in our study, but confirmation of accuracy can best be obtained with image guidance. This opens up avenues for further studies comparing pain relief between blind and ultrasound guided Suprascapular Nerve block and Intraarticular Steroid injections among hemiplegic and non-hemiplegic populations.

\section{CONCLUSION}

Suprascapular Nerve block has better efficacy as compared to Intra-articular steroid injection in patients with hemiplegic shoulder pain. Although intraarticular steroid injection has proven efficacy in shoulder pain resulting from inflammatory etiology, the multifactorial nature of hemiplegic shoulder pain means that a pain blocking intervention like suprascapular nerve block is more efficacious. This efficacy, coupled with low side effect profile of suprascapular nerve block makes it a suitable interventional procedure in the management of hemiplegic shoulder pain, especially in cases where steroid injections are contraindicated.

\section{Conflict of Interest: None.}

\section{Authors' Contribution}

MBN: Fomulatin of research question, conduct of study, data collection, AWB: Supervision of study, verification of results, WI: Statictical analysis of data, MR: Preparation of abstract \& conclusion.

\section{REFERENCES}

1. Wasay M, Khatri I, Kaul S. Stroke in South Asian countries. Nat Rev Neurol 2014; 10(3): 135-143.

2. Assad S, Zafar F, Tariq W, Shoaib R, Shah A. Frequency of ischemic stroke subtypes based on toast classification at a tertiary care center in Pakistan. Asian J Neur 2018; 13(4): 984-988.

3. Plecash A, Chebini A, Ip A, Lai J, Mattar A, Randhawa J et al. Updates in the Treatment of Post-Stroke Pain. Current Neurol Neurosci Rep 2019; 19(11): 401-404.

4. Westerlind E, Singh R, Persson H, Sunnerhagen K. Experienced pain after stroke: a cross-sectional 5-year follow-up study. BMC Neurol 2020; 20(1): 153-157.

5. Caglar N, Akin T, Aytekin E, Komut E, Ustabasioglu F, Okur S. Pain syndromes in hemiplegic patients and their effects on rehabilitation results. J Phys Ther Sci 2016; 28(3): 731-737.

6. Kalichman L, Ratmansky M: Underlying pathology and associated factors of hemiplegic shoulder pain. Am J Phys Med Rehab 2011; 90(9): 768-780.

7. Snels I, Beckerman H, Lankhorst GJ, Bouter LM. Treatment of hemiplegic shoulder pain in the Netherlands: results of a national survey. Clin Rehabilit 2000; 14(1): 20-27.

8. El-Badawy M. Suprascapular nerve block in the treatment of hemiplegic shoulder pain. Egyptian Rheumatol Rehab 2014; 41(1): 20-25. 


\section{Suprascapular Nerve Block Vs Intraarticular Steroid Injection}

9. Rah U, Yoon S, Moon D, Kwack K, Hong J, Lim Y, et al. Subacromial corticosteroid injection on poststroke hemiplegic shoulder pain: a randomized, triple-blind, placebo-controlled trial. Arch Phys Med Rehabilit 2012; 93(6): 949-956.

10. Jeon W, Park G, Jeong H, Sim Y. The Comparison of Effects of Suprascapular Nerve Block, Intra-articular Steroid Injection, and a Combination Therapy on Hemiplegic Shoulder Pain: Pilot Study. Ann Rehabilit Med 2014; 38(2): 167-73.

11. Sheikh SI, Ahmed A, Javaid S, Basit A, Sheikh IS, Sheikh SS, et al. Comparison of suprascapular nerve block \& intra-articular injection in the treatment of frozen shoulder. J Islam Int Med Coll 2012; 7(2): 76-81.

12. Smith T, Pappadis M, Krishnan S, Reistetter T. Stroke survivor and caregiver perspectives on post-stroke visual concerns and long-term consequences. Behav Neurol 2018; 2018(1): 1-8.

13. Cho C, Bae K, Kim D. Treatment strategy for frozen shoulder. Clin Orthop Surg 2019; 11(3): 249-255.

14. Kraal T, The B, Boer R, van den Borne M, Koenraadt K, Goossens $\mathrm{P}$, et al. Manipulation under anesthesia versus physiotherapy treatment in stage two of a frozen shoulder: a study protocol for a randomized controlled trial. BMC Musculoskelet Disord 2017; 18(1): 295-300.

15. Wang W, Shi M, Zhou C, Shi Z, Cai X, Lin T, et al. Effectiveness of corticosteroid injections in adhesive capsulitis of shoulder. Med 2017; 96(28): e7529.

16. El-Badawy M. Suprascapular nerve block in the treatment of hemiplegic shoulder pain. Egyptian Rheumatol Rehabil 2014; 41(1): 20-25.

17. Rah U, Yoon S, Moon D, Kwack K, Hong J, Lim Y, et al. Subacromial corticosteroid injection on poststroke hemiplegic shoulder pain: a randomized, triple-blind, placebo-controlled trial. Arch Phys Med Rehabil 2012; 93(6): 949-956.
18. Orhurhu V, Akinola O, Grandhi R, Urits I, Abd-Elsayed A Radiofrequency ablation for management of shoulder pain. Curr Pain Headache Rep 2019; 23(8): 51-54.

19. Sencan S, Celenlioglu A, Karadag-Sayg1 E, Midi İ, Gunduz O. Effects of fluoroscopy-guided intraarticular injection, suprascapular nerve block, and combination therapy in hemiplegic shoulder pain: a prospective double-blind, randomized clinical study. Neurol Sci 2019; 40(5): 939-946.

20. Lee KH, Khunadorn F. Painful shoulder in hemiplegic patients: a study of the suprascapular nerve. Arch Phys Med Rehabil 1986; 67(8): 818-820.

21. Yasar E, Vural D, Safaz I, Balaban B, Yilmaz B, Goktepe AS, et al Which treatment approach is better for hemiplegic shoulder pain in stroke patients: intra-articular steroid or suprascapular nerve block? A randomized controlled trial. Clin Rehabil 2011; 25(1): 60-68.

22. Ben-Aviv D, Boparai N, Wong D, Luu B, Kim E, Mitra R. The efficacy of suprascapular nerve block in treating shoulder pain. Arch Phys Med Rehabil 2007; 88(2): 67-71.

23. Idowu B, Ayoola O, Adetiloye V, Komolafe M. Sonographic evaluation of structural changes in post-stroke hemiplegic shoulders. Pol J Radiol 2017; 82(1): 141-148.

24. Fields B, Skalski M, Patel D, White E, Tomasian A, Gross J, et al. Adhesive capsulitis: review of imaging findings, pathophysiology, clinical presentation, and treatment options. Skeletal Radiol 2019; 48(8): 1171-1184.

25. Jung T, Lee S, Min S, Lee S, Yoo J. Does Combining a suprascapular nerve block with an intra-articular corticosteroid injection have an additive effect in the treatment of adhesive capsulitis? a comparison of functional outcomes after short-term and minimum 1-year follow-up. Orthopaedic J Sports Med 2019; 7(7) 232596711985927. 